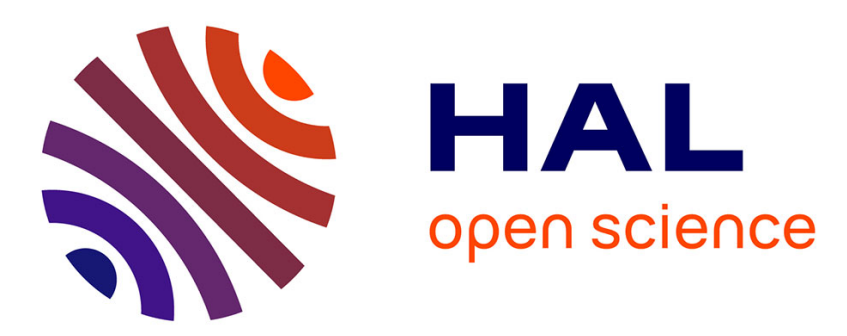

\title{
Modèles numériques pour le crash de structures composites complexes
}

\author{
Daniel Coutellier, Rémi Grenêche
}

\section{To cite this version:}

Daniel Coutellier, Rémi Grenêche. Modèles numériques pour le crash de structures composites complexes. Revue des composites et des matériaux avancés = Journal of Composite and Advanced Materials, 2007, 17 (1), pp.71-82. 10.3166/rcma.17.71-82 . hal-01789349

\section{HAL Id: hal-01789349 \\ https://hal.science/hal-01789349}

Submitted on 10 May 2018

HAL is a multi-disciplinary open access archive for the deposit and dissemination of scientific research documents, whether they are published or not. The documents may come from teaching and research institutions in France or abroad, or from public or private research centers.
L'archive ouverte pluridisciplinaire HAL, est destinée au dépôt et à la diffusion de documents scientifiques de niveau recherche, publiés ou non, émanant des établissements d'enseignement et de recherche français ou étrangers, des laboratoires publics ou privés. 


\title{
Modèles numériques pour le crash de structures composites complexes
}

\author{
Daniel COUTELLIER, Rémi GRENÊCHE
}

Equipe DRSC Commune ONERA/LAMIH

UMR CNRS 8530

Université de Valenciennes et du Hainaut Cambrésis

59313 Valenciennes Cedex 9

Mail : Daniel.Coutellier@univ-valenciennes.fr

Greneche_remi@yahoo.fr

RESUME. La performance dans le domaine de l'innovation industrielle passe inévitablement par le développement d'outils mieux adaptés aux nouvelles exigences. Le développement de méthodes de simulation numérique ou de techniques de réduction d'échelle s'inscrit dans cette optique puisqu'ils permettent de réduire les coûts liés aux campagnes expérimentales tout en augmentant la quantité et la qualité des informations qu'elles permettent d'obtenir. Cet article décrit la construction et l'exploitation d'un modèle par éléments finis permettant d'étudier un absorbeur d'énergie en composite stratifié sollicité en crash. Enfin, l'utilisation de ce modèle dans un contexte de similitude indirecte est discutée.

ABSTRACT. The performance in the field of the industrial innovation obviously passes through the development of tools more specifically adapted to the new requirements. The development of numerical simulation methods or scaling techniques is part of this procedure because they make it possible to reduce the costs related to experimental campaigns while increasing the quantity and the quality of the information which they permit to obtain. This article describes the construction and the exploitation of a finite elements model able to represent an energy absorber under crash loading. Lastly, the use of this model for the application with the indirect similarity method is discussed.

MOTS-CLÉS : matériau composite stratifié, éléments finis, modèle réduit, absorbeur d'énergie.

KEYWORDS: laminated composite material, finite elements, scale-down model, energy absorber. 


\section{Introduction}

La performance industrielle passe par une amélioration continue de l'ensemble des processus permettant le passage de la recherche et du développement à la conception de produits jusqu'à leur industrialisation y compris en intégrant leur maintenance et leur recyclage. La performance est aussi synonyme d'économie et donc de réduction des coûts, des délais et d'une augmentation de la qualité. D'autre part les cahiers des charges deviennent de plus en plus contraignants, ils évoluent en adéquation avec les préoccupations de la société surtout en termes de sécurité, de confort et d'efficacité. Les matériaux composites contribuent en partie à améliorer les performances, notamment en termes d'allègement des structures et de résistance aux sollicitations diverses.

Dans le domaine des transports aussi bien en aéronautique, qu'en automobile, ferroviaire ou naval, les matériaux non métalliques sont de plus en plus utilisés. En 1973, l'airbus A360B ne comprenait que 5\% de composite, en 1995 l'A330 et l'A340 en avait 15\%, en 2005 l'A380 en a plus de $25 \%$ et les objectifs dans 10 ans sont d'au moins $30 \%$ voire près de $50 \%$ sur certains types d'avion. Dans le domaine des loisirs et du sport, les composites jouent là aussi un rôle majeur, ils facilitent l'emploi des produits et contribuent à l'amélioration des performances de l'utilisateur.

Quel que soit le domaine d'application, ces matériaux sont de plus en plus utilisés, mais leurs complexités engendrent la nécessité de réaliser de nombreux tests pour valider les orientations prises lors de la conception des produits, en effet les comportements sont dépendants de nombreux phénomènes tels que la constitution et la répartition des composants, les procédés de fabrication souvent encore artisanaux, les types de sollicitations, et les modes de déformation sont très souvent complexes, notamment quand il s'agit de déformation irréversible.

L'innovation joue un rôle fondamental pour modifier et améliorer la conception, elle permet une définition des processus de plus en plus performante. Néanmoins le passage obligatoire avant tout, est bien évidemment l'amélioration des différents outils utilisés à un moment ou à un autre dans l'ensemble des étapes de tous les processus employés. Entre autres, l'amélioration des outils de simulation numérique favorise le développement de la conception de nouvelles structures. L'article propose une revue des possibilités et des facteurs primordiaux intervenant dans la simulation numérique de matériaux stratifiés avec une application plus particulière à un absorbeur d'énergie sollicité dynamiquement en compression axiale et étudié à différentes échelles.

Parmi l'ensemble des modèles permettant la simulation numérique des composites stratifiés, nous avons porté une attention particulière sur 2 modèles, l'un appelé bi-phase développé par ESI Group et l'autre appelé méso-modèle développé par Ladevèze. Ces deux modèles sont disponibles dans Pam-Crash ${ }^{\mathrm{TM}}$, outil de simulation utilisée pour nos développements. Le premier modèle a été développé pour représenter le comportement au crash d'une structure automobile, l'autre a été 
intégré par le LAMIH dans le cadre de plusieurs thèses pour représenter le comportement de multi-matériaux comme le GLARE ou autres stratifiés verre Eépoxy ou carbone-époxy. Les développements ont portés sur l'intégration de nombreux phénomènes comme les effets de vitesse très importants dans le domaine du crash et l'intégration du délaminage dans le cadre de méthodologies couplées. Plusieurs exemples ont illustrés la pertinence des développements. Une présentation de ces développements est disponible dans plusieurs articles (Coutellier et al., 1998) (Coutellier et al., 2002) (Coutellier et al., 2004) (Rozycki et al., 2002). Ici une nouvelle approche à partir de modèles 3D est proposée pour représenter la phénoménologie rencontrée lors de la compression axiale en dynamique rapide d'absorbeurs d'énergie représentés à 3 échelles différentes. Une comparaison avec des résultats issus d'une campagne expérimentale illustre la pertinence de cette nouvelle approche basée sur la prise en compte des contacts et frottements dans les interfaces des modèles numériques.

\section{Réduction d'échelle sur structure stratifiée}

L'application de méthodes de réduction d'échelle pose de nombreux problèmes dans des cas aussi complexes que le crash d'absorbeurs d'énergie fabriqués en composite stratifié (Grenêche et al., 2005) (Dormegnie, 2001). En effet, l'utilisation du théorème des $\pi$ de Vaschy-Buckingham est gênée par le très grand nombre de paramètres nécessaires à la caractérisation du comportement des matériaux nonhomogènes anisotropes et ne permet de prendre en compte les effets des distorsions qui sont inévitables. La méthode directe (Trompille, 2003) (Simitses, 2001) nécessite un modèle analytique capable de représenter fidèlement tous les phénomènes présents lors de l'essai. Or, certains phénomènes comme le délaminage ou l'influence de la vitesse de déformation sont encore mal connus (Rozycki, 2000). Enfin, la similitude indirecte (Dacheux, 1993) (Langle, 1997) demande de construire un modèle par éléments finis suffisamment fiable pour reproduire tous les phénomènes mis en jeu. Mais, malgré les progrès constants des codes de calcul, de tels modèles restent difficiles à construire notamment pour l'application aux matériaux composites sollicités en dynamique rapide.

Pour contourner ces difficultés, une nouvelle méthode à été proposée (Grenêche et al., 2005) en s'appuyant sur le cas d'absorbeurs d'énergie de forme oméga en verre E/époxy constitués de plis à $+/-45^{\circ}$ uniquement. Elle consiste à réaliser une interpolation polynomiale sur des résultats d'essais de maquettes. Puis, le polynôme obtenu [1] est utilisé dans la méthode directe. Pour les absorbeurs oméga sollicités en quasi-statique, le polynôme déterminé est (Grenêche et al., 2005) :

$$
\mathrm{F}_{\mathrm{pal}}=\mathrm{An} \beta^{2}+\mathrm{B} \beta-\mathrm{C}
$$

Avec $\mathrm{F}_{\mathrm{pal}}$ la valeur moyenne du palier d'effort, $\mathrm{n}$ le nombre d'interfaces entre plis d'orientations différentes, $\beta$ le facteur d'échelle géométrique, $\mathrm{A}, \mathrm{B}$ et $\mathrm{C}$ des coefficients déterminés expérimentalement. 
Mais cette méthode est limitée à des empilements présentant peu de plis d'orientations différentes car leur nombre fait augmenter le nombre de coefficients du polynôme et donc le nombre d'essais nécessaires pour les identifier. Face à cet inconvénient, un modèle E.F. a été créé de façon à permettre une amélioration des connaissances du comportement de la structure en étudiant les paramètres de manière indépendante dans le but d'identifier un à un les termes du polynôme et ainsi obtenir une formulation plus analytique. Une fois validé, ce modèle E.F., qui est présenté dans la suite de cet article, pourrait aussi permettre l'application de la similitude indirecte.

\section{Construction du modèle}

\subsection{Description des essais}

La structure étudiée est un absorbeur d'énergie à section oméga (Figure 1) réalisé par moulage de pré-imprégné en verre E/époxy semblable à ceux utilisés en aéronautique. Les empilements sont constitués uniquement de plis à $+/-45^{\circ}$ de façon à ce que le mode de ruine en fragmentation soit négligeable devant le mode de ruine en ouverture interlaminaire (Farley et al., 1992) (Hull, 1991). Trois échelles de cette structure ont été testées en compression axiale à $5 \mathrm{~mm} / \mathrm{s}$. Les échelles $1 / 2$ et 1 sont dimensionnées en appliquant une amplification de la structure à échelle 1/4 par une similitude géométrique. Les conditions d'essais sont mises à l'échelle par la similitude de Cauchy (Dormegnie, 2001). Enfin, les séquences d'empilement sont modifiées pour permettre une mise à l'échelle de l'épaisseur du stratifié en utilisant les techniques de réagencement de plis proposées par Jackson (Jackson, 1994), ce qui permet d'obtenir trois configurations différentes pour l'échelle $1 / 2$ et cinq pour l'échelle 1 tout en conservant des répartitions d'interfaces comparables.

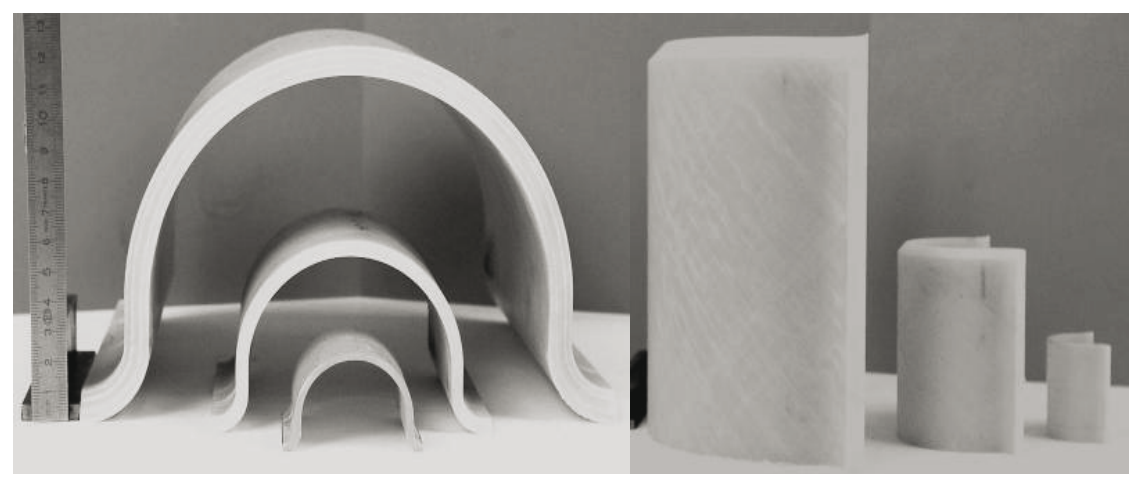

Figure 1 : Eprouvettes oméga vues dessus et de coté.

\subsection{Phénomènes mis en jeu}

Les absorbeurs d'énergie en composite stratifié présentent une ruine par rupture fragile qui permet une absorption d'énergie supérieure aux métaux qui absorbent l'énergie par déformation plastique. Lorsque la ruine de la structure se fait essentiellement en ouverture interlaminaire, comme dans le cas étudié ici, la 
première rupture se produit au niveau de l'interface interlaminaire médiane. Les plis fléchissent alors sous l'effet de l'impacteur, formant deux branches qui délaminent en cisaillement. Ces interfaces délaminées sont alors le lieu de frottements. Si la structure présente un rayon de courbure, la flexion des branches entraîne aussi la formation d'un nombre élevé de fissures longitudinales découpant les plis en bandes. Mais la majorité des auteurs (Mamalis et al., 1998) (Hull, 1991) s'accordent à dire que cette fissuration ne représente que quelques pourcents de l'énergie totale absorbée. Enfin, Hull (Hull, 1991) a montré que les frottements entre l'impacteur et l'éprouvette absorbent aussi une énergie non négligeable quel que soit l'état de surface de l'impacteur. L'énergie semble donc être essentiellement absorbée par la flexion des plis et les différents frottements. Une modélisation fiable passe donc par une représentation correcte de tous ces phénomènes.

\subsection{Construction du modèle}

\subsubsection{Préambule}

Contrairement à ce qui se trouve classiquement dans la littérature (Bisagni et al., 2005) (Mac Carthy et al., 2001), le modèle E.F. est construit à partir d'éléments volumiques car les modèles réalisés à partir d'éléments coques multi-couches ne permettent pas d'introduire de frottements dans les interfaces délaminées, les mouvements relatifs des plis n'étant pas pris en compte. Le modèle de pli utilisé est le modèle biphase disponible dans le code de calcul PAM-CRASH ${ }^{\mathrm{TM}}$. Les interfaces entre plis sont modélisées par l'intermédiaire d'un matériau «lien » permettant de relier la surface d'un pli aux nœuds du pli adjacent. Le délaminage est alors introduit en utilisant un critère de rupture de type cercle de Mohr.

Une première modélisation est réalisée sur une «tranche » d'oméga (Figure : 2) de façon à valider la capacité à délaminer du modèle, à déterminer les valeurs des contraintes à introduire dans le critère rupture et à vérifier la réponse en flexion des plis. Les premières simulations ayant été réalisées sans introduction de frottement, les éprouvettes fléchissaient intégralement du même coté. L'introduction de frottements de Coulomb entre l'impacteur et la structure a permis de pallier ce problème.

a)

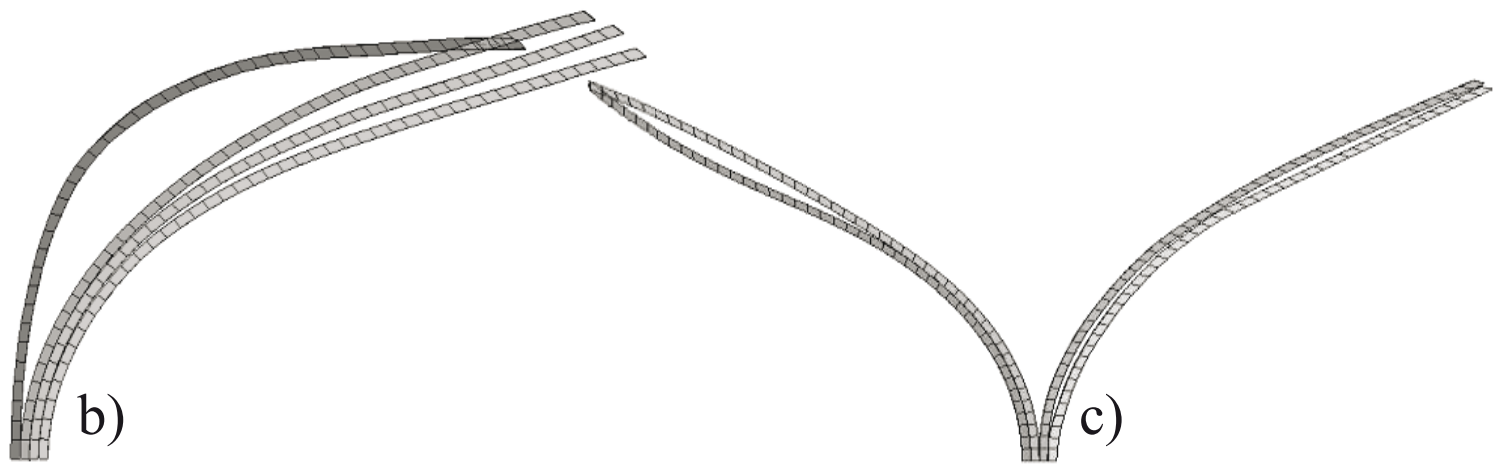

Figure 2 : Modélisation d'une tranche d'oméga : a) état initial ; b) état final sans frottements ; c) état final avec frottements. 
N'ayant pas réalisé d'essais de caractérisation de ces frottements, la valeur du coefficient a été déterminée en recalant les résultats des simulations sur des résultats d'essais de compression. Des valeurs de paliers d'effort encourageantes (même ordre de grandeur que celles attendues) ont été obtenues pour un coefficient de frottement de 0,5 .

\subsubsection{Modélisation de l'échelle 1/4}

Une fois cette pré-étude achevée, une simulation des éprouvettes à l'échelle $1 / 4$ a été réalisée. La fissuration longitudinale n'absorbant qu'une faible part de l'énergie et étant délicate à intégrer, il a été décidé de ne pas la représenter. Par conséquent, la déformation globale obtenue est différente de celle observée expérimentalement: sans cette fissuration, les plis extérieurs ne peuvent que fléchir vers l'intérieur de l'éprouvette (Figure 3).
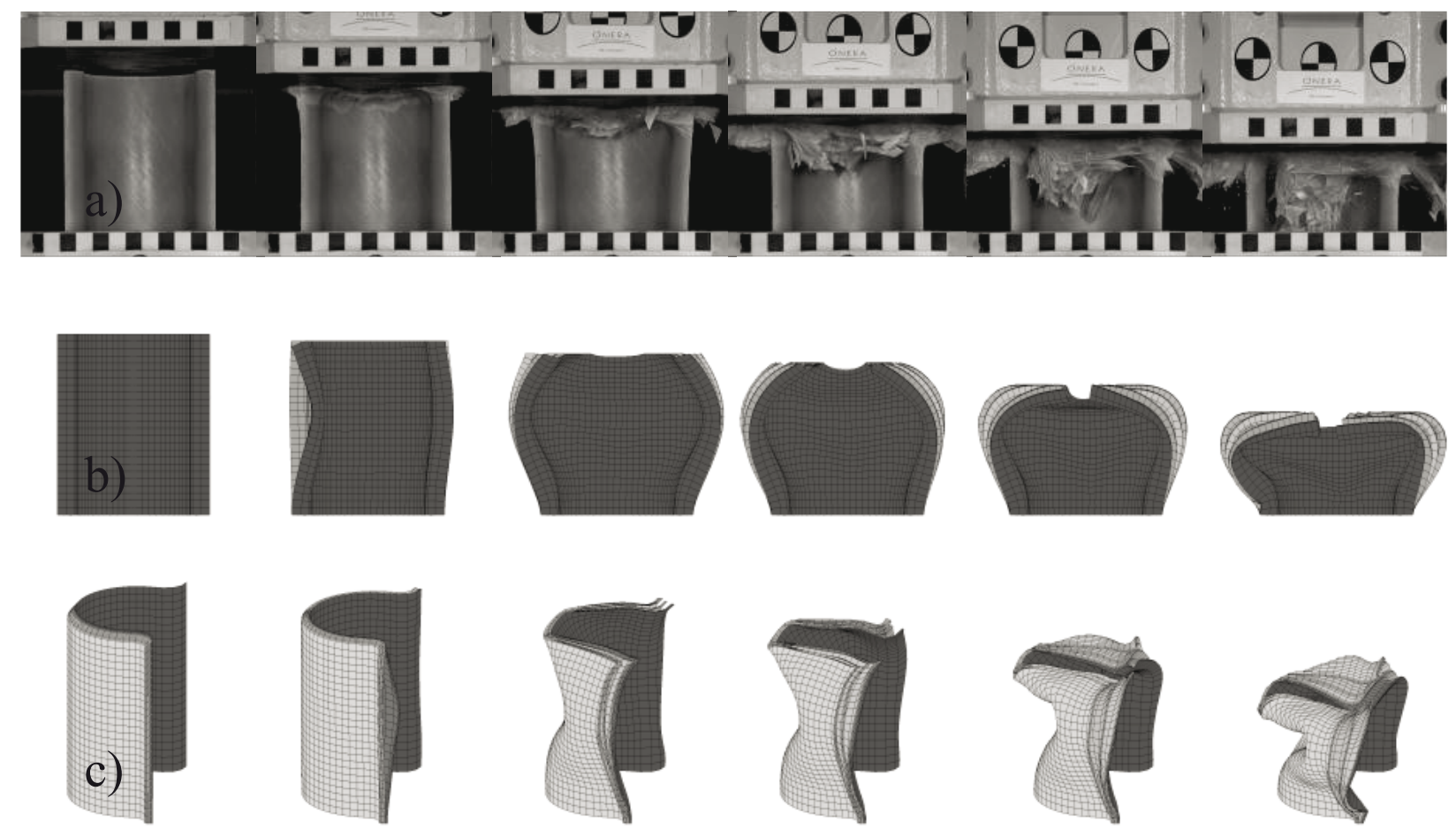

Figure $3:$ a) éprouvette à l'échelle 1 en cours d'essais ; b) déformation du modèle numérique vue de face et c) en vue iso.

Des frottements de type Coulomb ont été introduits au niveau de toutes les interfaces qui délaminent, mis à part l'interface médiane puisque les plis qui la composent fléchissent expérimentalement dans des directions opposées. Le coefficient a aussi été déterminé en recalant les résultats des simulations à l'aide de résultats d'essais. Les paramètres des interfaces et les deux coefficients de frottements ayant des influences comparables sur la valeur de l'effort de palier et le temps de calcul avoisinant 4 jours en monoprocesseur, l'utilisation d'un logiciel d'optimisation n'a pu être envisagée. Le recalage est donc resté approximatif. Toutefois les résultats ont été jugés suffisamment satisfaisants (Figure 4) pour tenter de valider la similitude indirecte en modélisant les échelles supérieures. 


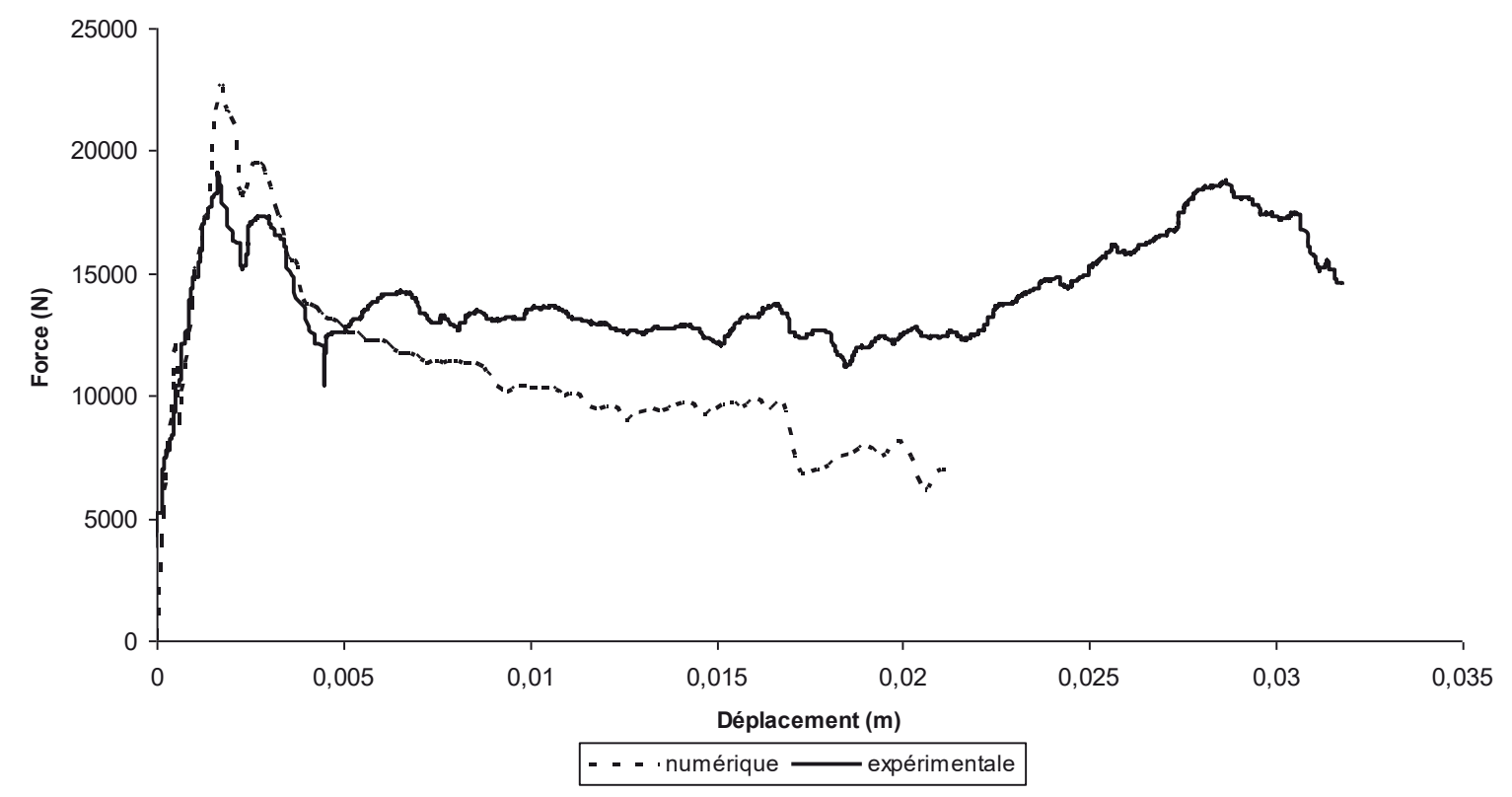

pour l'échelle $1 / 4$.

\section{Exploitation du modèle}

\subsection{Modélisation des éprouvettes à échelle 1/2}

Les simulations des trois configurations à échelle $1 / 2$ sont réalisées sans modifier les paramètres déterminés lors de l'étude de l'échelle 1/4. Quelle que soit la configuration, les niveaux d'effort restent comparables aux résultats expérimentaux comme le montre le graphe 5 et leur évolution avec le nombre d'interfaces semble correctement modélisée.

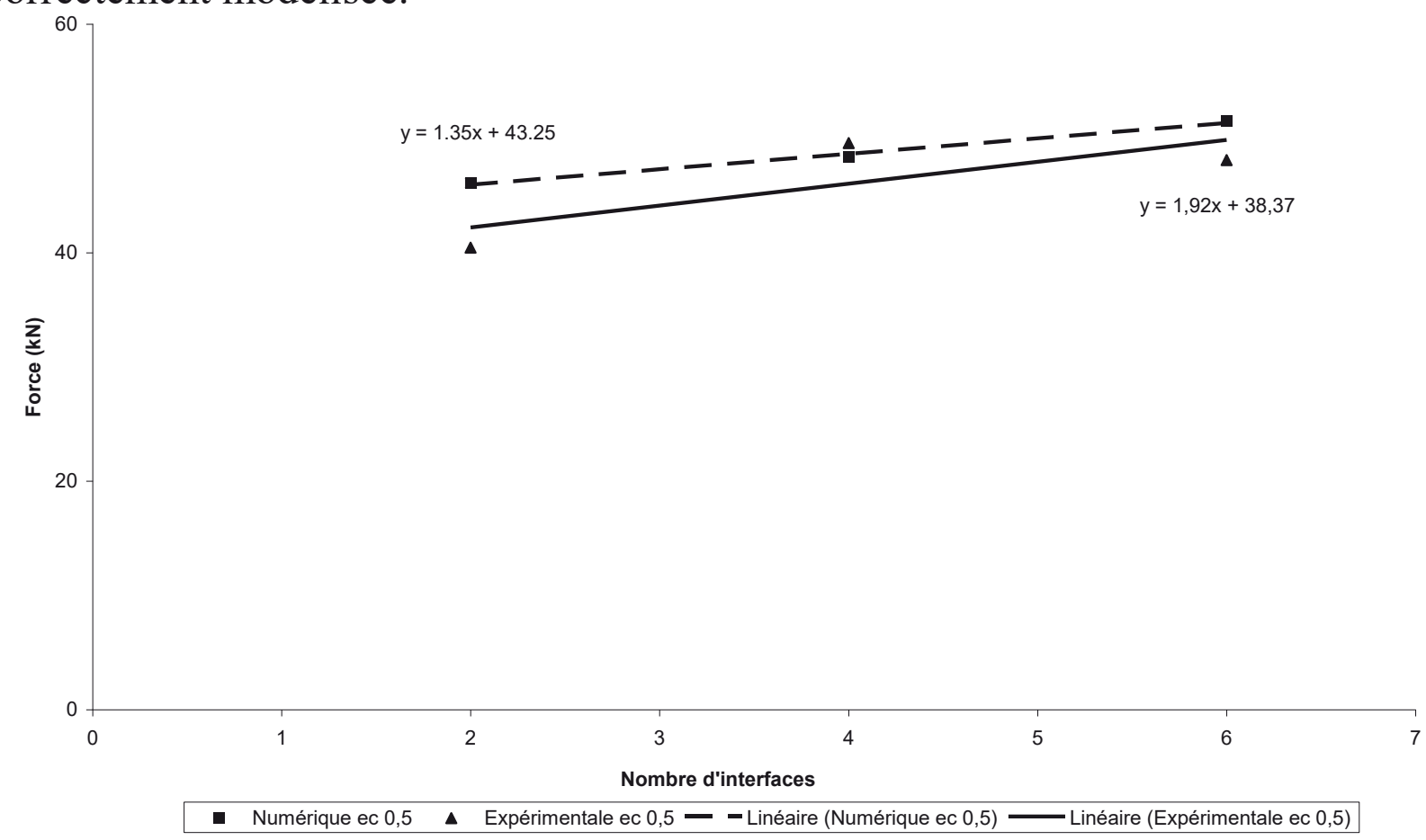

Figue 5 : Comparaison des courbes effort de palier moyen/nombre d'interfaces obtenues numériquement et expérimentalement pour des omégas échelle 1/2. 
De plus, comme lors des essais expérimentaux, il a été observé que seules les interfaces entre plis d'orientations différentes sont sujettes au délaminage.

Toutefois, le passage à l'échelle $1 / 2$ ne peut valider qu'en partie la similitude indirecte : en effet, mis à part le nombre d'interfaces, l'influence des effets d'échelle observés expérimentalement (termes du polynôme qui n'ont pas $\beta^{2}$ en facteur) reste relativement limitée lors du passage de l'échelle $1 / 4$ vers l'échelle $1 / 2$. Une simulation d'éprouvette à l'échelle 1 est donc nécessaire pour finir de valider la similitude indirecte en vérifiant que l'effet d'échelle lié à la résistance en flexion des plis est bien représenté.

\subsection{Modélisation des éprouvettes à échelle 1}

Pour permettre une réduction du nombre d'éléments nécessaires à la modélisation, seule les configurations d'éprouvettes à échelle 1 présentant des plis doublés ont été modélisées (les temps de calcul pour l'échelle 1/2 dépassant les 11 jours). Comme pour l'échelle $1 / 4$, l'influence du nombre d'interfaces correspond aux résultats expérimentaux. Par contre, les valeurs moyennes des paliers d'effort sont environ deux fois supérieures à celles mesurées lors des essais. La figure 6 met en évidence le fait que ce facteur 2 ne semble pas concerner le terme lié au nombre d'interfaces. Il n'est donc pas lié au délaminage ou aux frottements entre plis, mais affecte la résistance en flexion des plis.

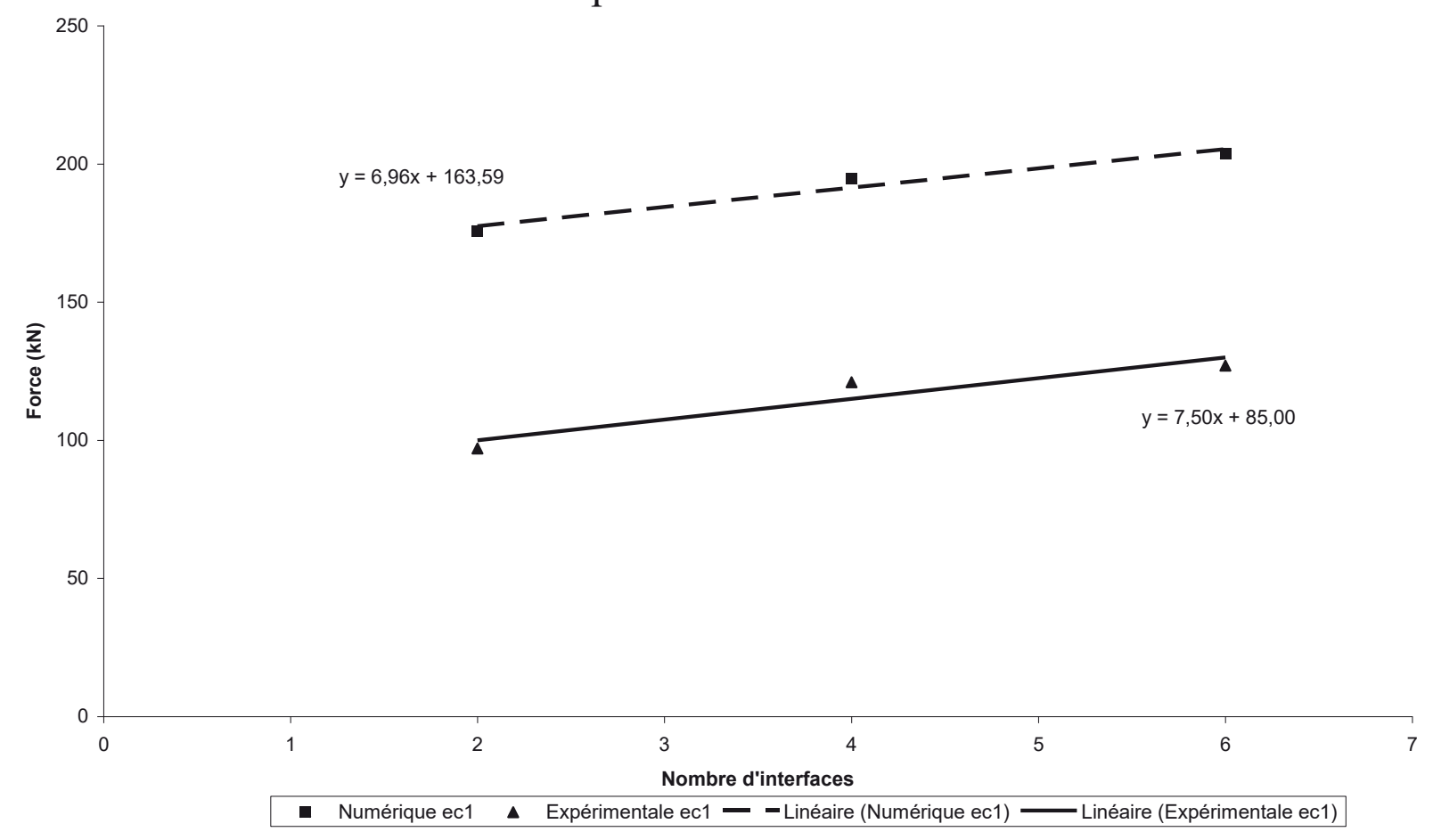

Figue 6 : Comparaison des courbes effort de palier moyen/nombre d'interfaces obtenues numériquement et expérimentalement pour des omégas échelle 1.

L'effet d'échelle lié à la résistance en flexion des plis n'est donc pas correctement modélisé. Cet effet d'échelle est donc lié à un phénomène non ou mal représenté qui, de plus, est lié à la résistance en flexion des plis. L'hypothèse la plus probable est donc que la fissuration longitudinale possède une influence plus 
importante sur l'effort de palier que ce qui est classiquement décrit dans la littérature. Des mesures post-mortem ont donc été réalisées sur les éprouvettes des différentes échelles. Les résultats de ces mesures (Tableau 1 et Figure 7) mettent effectivement en évidence un effet d'échelle puisque les largeurs des bandes créées par la fissuration longitudinale n'évoluent pas selon le facteur d'échelle géométrique. L'utilisation du modèle EF pour utiliser la similitude indirecte passe donc par l'intégration de la fissuration longitudinale.

\begin{tabular}{|c|c|c|c|}
\hline $\begin{array}{c}\text { Echelle de } \\
\text { l'oméga }\end{array}$ & $1 / 4$ & $1 / 2$ & 1 \\
\hline $\begin{array}{c}\text { Largeur moyenne } \\
\text { L des bandes } \\
(\mathrm{mm})\end{array}$ & 2,9 & 6,4 & 19,6 \\
\hline
\end{tabular}

Tableau 1 : Largeur des bandes créées par la fissuration longitudinales.

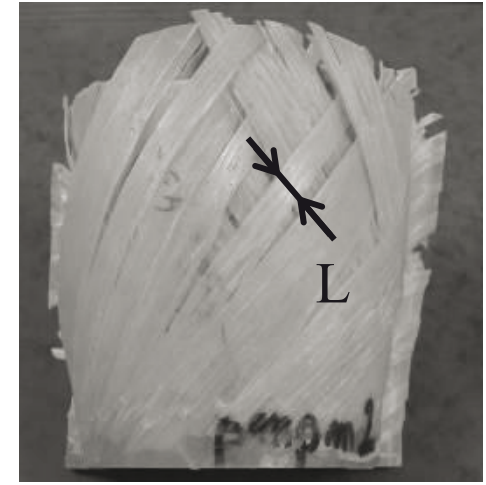

Figue 7 : Bandes formées par la fissuration longitudinale.

\subsection{Comparaison avec la littérature}

Enfin, pour que ce modèle puisse avoir réellement un sens physique, il est nécessaire de vérifier si les valeurs des paramètres déterminés numériquement sont en accord avec les valeurs répertoriées dans la littérature. Le tableau 2 présente une comparaison entre les valeurs numériques et des valeurs mesurées pour des matériaux et des géométries relativement proches de ceux utilisés lors de cette étude.

Les valeurs déterminées numériquement sont donc en parfait accord avec les valeurs mesurées par d'autres auteurs, même si les intervalles sont relativement larges, du fait de la diversité de structures et de matériaux étudiés. Toutefois, cette comparaison permet de valider le sens physique des valeurs déterminées puisque les ordres de grandeur correspondent.

De plus, un essai de type ARCAN est en cours de développement en collaboration avec l'ONERA (Delsart et al., 2005) afin de mesurer les caractéristiques des interfaces. Les premiers résultats obtenus pour le même matériau donnent une contrainte maximale en cisaillement de $29 \mathrm{MPa}$ tout à fait en accord avec les valeurs issues des simulations numériques et $22 \mathrm{MPa}$ pour la contrainte maximale en arrachement ce qui est inférieur à la valeur déterminée numériquement, mais des mesures par corrélation d'images ont mis en évidence des non-uniformités du champ de contraintes lors de ces essais pouvant expliquer cette sous-évaluation de la contrainte maximale. 


\begin{tabular}{|c|c|c|c|}
\hline Paramètres & $\begin{array}{c}\text { Valeurs déterminées } \\
\text { numériquement }\end{array}$ & $\begin{array}{c}\text { Valeurs issues de } \\
\text { la littérature }\end{array}$ & Références \\
\hline $\begin{array}{c}\text { Contrainte de } \\
\text { rupture des } \\
\text { interfaces }+/-45 \text { en } \\
\text { arrachement }\end{array}$ & $60 \mathrm{MPa}$ & $55-75 \mathrm{MPa}$ & $\begin{array}{c}\text { Gornet et al. 2000, } \\
\text { Lifshitz et al. 1998 }\end{array}$ \\
\hline $\begin{array}{c}\text { Contrainte de } \\
\text { rupture des } \\
\text { interfaces }+/-45^{\circ} \text { en } \\
\text { cisaillement }\end{array}$ & $30 \mathrm{MPa}$ & $23-48 \mathrm{MPa}$ & Daniel et al. 1999 \\
\hline $\begin{array}{c}\text { Coefficient de } \\
\text { frottement } \\
\text { acier/époxy }\end{array}$ & 0,35 & $0,23-0,49$ & $\begin{array}{c}\text { Mamalis et al. } \\
1998, \text { Hull 1991 }\end{array}$ \\
\hline $\begin{array}{c}\text { Coefficient de } \\
\text { frottement entre } \\
\text { plis à }+/-45^{\circ}\end{array}$ & 0,50 & $0,38-0,66$ & $\begin{array}{c}\text { Mamalis et al. } \\
1998, \text { Schön 2000 }\end{array}$ \\
\hline
\end{tabular}

Tableau 2 : Comparaison des valeurs déterminées numériquement avec celles issues de la littérature.

Enfin, en annulant successivement certains paramètres, les participations respectives à l'absorption d'énergie de quelques phénomènes ont pu être déterminées. Ainsi, pour les éprouvettes à l'échelle 1/2, les frottements impacteur / structure absorbent environ 35\% de l'énergie totale et les frottements entre plis adjacents (après délaminage) absorbent de $3 \%$ pour celles à 2 interfaces à $13 \%$ pour celles à 6 interfaces. A titre de comparaison, d'autres auteurs (Mamalis et al., 1998) annoncent entre 50 et $55 \%$ de l'énergie totale absorbée par les frottements (sans distinction). Donc, encore une fois, les résultats obtenus numériquement sont en accord avec la littérature.

\section{Conclusions et perspectives}

En résumé, un modèle E.F. a été construit afin de permettre de découpler certains phénomènes pour faciliter l'étude du crash d'absorbeur d'énergie en composite stratifié. Cette modélisation, construite par une approche phénoménologique, se distingue par le fait qu'elle n'utilise pas un élément coque multicouche mais un élément volumique, ce qui permet de modéliser de façon plus précise certains phénomènes comme les frottements entre plis.

Les résultats sont très encourageants et assez réalistes malgré le fait qu'un recalage précis n'ait pu être effectué. Mais ce problème pourrait être résolu en mesurant les coefficients de frottements (ce qui nécessite la conception d'un montage spécifique) et les caractéristiques des interfaces. Pour ce dernier point, un essai de type ARCAN (Delsart et al., 2005) a été développé en collaboration avec l'ONERA Centre de Lille.

Les résultats obtenus ont permis de quantifier la participation à l'absorption totale d'énergie de quelques phénomènes comme les frottements, ce qui pourrait 
permettre d'identifier certains des termes du polynôme [1] en réalisant des études variationnelles.

Enfin, une comparaison entre les résultats numériques et expérimentaux a permis de détecter un effet d'échelle qui apparaît au niveau de la fissuration longitudinale. La non modélisation de ce type de fissuration empêche la prise en compte de cet effet d'échelle dans les résultats, ce qui limite l'utilisation de la similitude indirecte. Un modèle numérique de fissuration longitudinale est en cours de construction, il sera néanmoins difficile de l'intégrer dans la phase de conception de nouvelles structures du fait de la taille importante de la modélisation.

\section{Bibliographie}

Bisagni C., DI Pietro G., Fraschini L, Terletti D., «Progressive crushing of fiber-reinforced composite structural components of a formula one racing car », Composite Structures 68, Elsevier Science, p 491-503, 2005.

Coutellier, D., Gauthier, C., Ravalard, Y., Ni, X. \& Haug, E., « Un élément multi-matériaux pour l'étude du comportement en crash des structures stratifiées : synthèse et perspectives» Revue Européenne des Eléments Finis, Vol. 7. n 1/2/3, (pp.177-192), 1998.

Coutellier D., Rozycki, P., " New functionalities for PAMCRASH ${ }^{\mathrm{TM}}$ multi-layered multimaterial element », Composites PartA - Applied Science and Manufacturing, 31, p 841-851, 2000.

Coutellier D., Rozycki P., « Mise en évidence de la sensibilité à la vitesse de déformation des composites unidirectionnels verre - époxy : proposition d'un modèle numérique ", Revue des Composites et Matériaux Avancés Vol.14-1, p 31-50, 2004.

Dacheux F., Développement d'une technique de similitude indirecte sur modèles réduits ; Application à l'étude du comportement en collision des véhicules de transport guidé, Thèse de doctorat, Université de Valenciennes et du Hainaut Cambrésis, 1993.

Daniel L., Hogg P.J., Curtis P.T., « The relative effects of through-thickness properties and fibre orientation on energy absorption by continuous fibre composites », Composites Part B 30, Elsevier Science, p 257-266, 1999.

Delsart D., Mortier J. M., Dagois M., « Experimental characterization and modelling of the inter-ply interface properties of fibre reinforced composite materials », International Conference on Impact Loading of Lightweight Structures, Florianópolis - Brazil, 2005.

Dormegnie D., Contribution à l'étude de lois de similitude applicables au crash de structures composites stratifiées du type absorbeur d'énergie, Thèse de doctorat, Université de Valenciennes et du Hainaut Cambrésis, 2001.

Farley G.L., Jones R.M., « Crushing characteristics of continuous fiber-reinforced composite tubes », Journal of Composite Materials 26, Technomic Publishing, p 37-50, 1992.

Farley G.L., Jones R.M., «Prediction on the energy-absorption capability of composite tubes », Journal of Composite Materials 26, Technomic Publishing, p 388-404, 1992.

Gornet L, Lévêque D., Perret L., «Modélisation, Identification et simulation éléments finis des phénomènes de délaminage dans les structures composites stratifiées ", Mécanique et Industries 1, Elsevier Science, p267-276, 2000. 
Grenêche R., Coutellier D., Ravalard Y., «Utilisation de modèles réduits pour essais de crash de composites stratifiés », $14^{\text {ème }}$ Journées Nationales sur les Composites, Compiègne, 2005.

Grenêche R., Coutellier D., Ravalard Y., « Nouvelle méthodologie de dimensionnement de

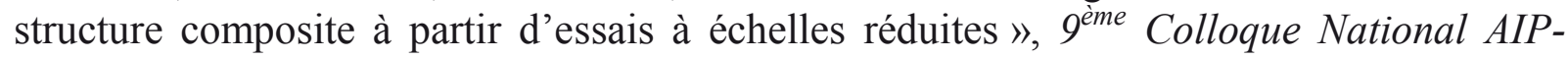
PRIMECA, La Plagne, 2005.

Hull D., " A unified approach to progressive crushing of fibre-reinforced composite tubes », Composite Science and Technology 40, Elsevier Science, p 377-421, 1991.

Jackson K.E., Workshop and scaling effects on composite materials and structures, Rapport NASA, Langley Research Center Hampton Virginia, NASA conference publication 3271, 1994.

Langle F., Contribution à l'élaboration de design de substitution en similitude indirecte sur modèles réduits; Application à l'étude du comportement en collision des absorbeurs axiaux, Thèse de doctorat, Université de Valenciennes et du Hainaut Cambrésis, 1997.

LifshitzJ. M., Leber H., « Response of fiber-reinforced polymer to high strain-rate loading in interlaminar tension and combined tension/shear », Composite Science and Technology 58, Elsevier Science, p987-996, 1998.

Mac Carthy M.A., Wiggenraad J.F.M., « Numerical investigation of a crash test of a composite helicopter subfloor structure », Composite Structures 51, Elsevier Science, p 345-359, 2001.

Mamalis A.G., Manolakos D.E., Demosthenous G.A., Ioannidis M.B., Crashwothiness of composite thin-wall structural components, Technomic Publishing CO. INC., 1998.

Rozycki P., Contribution au développement de lois de comportement pour matériaux composites soumis à l'impact, Thèse de doctorat, Université de Valenciennes et du Hainaut Cambrésis, 2000.

Rozycki P., Coutellier, D., « Sensibilité à la vitesse de déformation des matériaux composites unidirectionnels », In Hermes (Eds.) Revue Européenne des Eléments Finis, Vol 11 № 23-4, p 263-276, 2002.

Schön J., « Coefficient of friction of composite delamination surfaces », Wear 237, Elsevier Science, p 77-89, 2000.

Sinitses G.J., «Structural similitude for flat laminated surfaces », Composite Structures 51, Elsevier Science, p 191-194, 2001.

Trompille V., Etude expérimentale et théorique du comportement d'un tunnel renforcé par boulonnage frontal, Thèse de doctorat, INSA de Lyon, 2003.

\section{Remerciements}

La Région Nord-pas-de-Calais, le GRRT, l'ONERA, le FEDER, la société ESI Group et le CNRS sont ici remerciés pour leur soutien respectif à ces travaux. 